Saudi Journal of Medicine

Abbreviated Key Title: Saudi J Med ISSN 2518-3389 (Print) |ISSN 2518-3397 (Online)

\title{
Role of Vascular Ultrasonography in COVID-19-- Review Article
}

\author{
Nazeeha Waseem ${ }^{1 *}$, Beenish Abbas ${ }^{2}$, Usman Nazir $^{3}$, Hafsa Waseem ${ }^{4}$, Abid Ali ${ }^{5}$, Taiba Zulfiqar ${ }^{6}$ \\ ${ }^{1}$ Medical Imaging Doctor, Department of Radiological Sciences and Medical Imaging, the University of Lahore, Gujrat, Pakistan \\ ${ }^{2}$ Radiography Technologist, Government College University, Faisalabad, Pakistan \\ ${ }^{3}$ Microbiologist, Government College University, Faisalabad \\ ${ }^{4}$ Senior lecturer, Department of Microbiology, Fazaia Medical College, Islamabad, Pakistan \\ ${ }^{5}$ Associate Professor, Department of Allied Health Sciences, the University of Lahore, Gujrat, Pakistan \\ ${ }^{6}$ Senior Lecturer, Department of Radiological Sciences and Medical Imaging University of Lahore Gujrat, Pakistan
}

DOI: $10.36348 /$ sjm.2021.v06i02.002 $\quad$ | Received: 11.02.2021 | Accepted: 23.02.2021 | Published: 26.02 .2021

*Corresponding Author: Nazeeha Waseem

\section{Abstract}

Corona virus pandemic has certainly become one of the most challenging diseases ever to come to light in the history of humankind affecting from individual level to national level worldwide affecting every aspect of life. To decrease the mortality rate in coronavirus patients, the most significant role is played by early interventions when the virus is in its initial stages. To achieve this, various imaging techniques are employed out of which vascular ultrasonography is one of the most important ones. Although a little light has been shed so far on the mechanisms regarding the coagulopathies caused by Corona virus, it is without a doubt one of the hottest topics in the scientific community these days. The virus enters the body by interacting with angiotensin converting enzyme receptors which are present in different areas of the body like lungs, heart, arteries, and kidneys. The virus Interacts with endothelial cells becoming a cause for their activation and consequently initiating the coagulation system. Arterial thrombosis, deep venous thrombosis, acute respiratory distress syndrome, sepsis-induced coagulopathy, etc. are the complications caused by the virus and are the reason for mortality in severe cases of coronavirus. Vascular ultrasonography enables us to stop the virus in its tracks long before it can become fatal, ultimately helping us in decreasing the mortality rate significantly.

Keywords: Covid-19, Vascular Ultrasound, Thrombosis, Coagulopathy.

Copyright (C) 2021 The Author(s): This is an open-access article distributed under the terms of the Creative Commons Attribution 4.0 International License (CC BY-NC 4.0) which permits unrestricted use, distribution, and reproduction in any medium for non-commercial use provided the original author and source are credited.

\section{INTRODUCTION}

Corona virus 19 is one of the biggest challenges humanity is facing right now. Coronavirus disease caused by the novel coronavirus 2019 has posed a horrifying threat against the very existence of the human race $[1,2]$. Mild to moderate symptoms appear in almost every age group in this disease. However, the age group that is most susceptible to death by coronavirus are the elderly ( $>$ than 65 years of age) [3] or people with some underlying disease [4]. An impressive high transmission rate of this virus has made it very difficult for us to control its infectivity [5]. The fact that it affects so many people in such a little time plays a vitally important role in causing the pandemic. Two major factors contribute to the critical conditions of the patient suffering from SARS-COV2 out of which the first one is the cytopathic effect [6] and the other factor is the storm of cytokines caused by this virus. The clinical spectrum of this disease is very variable including many complications like acute respiratory distress syndrome, septic shock, metabolic acidosis and multiple organ failure $[7,8]$.

It has been formulated that there is a strong association between vascular abnormalities and the stage of the disease. The more severe the disease, the more thromboembolic events are reported. Evidence provided by recent studies suggests that coagulation disorders are one of the most important complications of coronavirus. Although very little data has been gathered so far regarding the mechanisms behind coagulative complications associated with coronavirus but the importance of this cannot be neglected [9]. Detecting these disorders in their early stages is very beneficial in our battle against this disease. Imaging techniques like CT scan, MRI scan, vascular ultrasound, and lungs ultrasound $[10,11]$ are employed to find out the presence of venous and arterial thrombi or tumors enabling us to intervene in the progression of the disease long before it can cause complications. 
Vascular ultrasound gives us a pretty good picture of the progression of the disease and tells us whether the viral disease is in the early or late stage. Understandably, a good diagnosis with early interventions helps in resolving the complications of the disease. The mortality rate reported after early interventions to be very low as compared to late diagnosis and late interventions in the progression of the disease $[12,13]$. The virus after entering the body causes damage to endothelial cells resulting in exposure of sub endothelial collagen activating the coagulation cascade. When a clot breaks, fibrin degradation products and D-dimers are seen. In coronavirus patients who suffered death as a result of thromboembolic events, elevated levels of D-Dimers, and fibrin degradation products were seen. The increased level of D-Dimers in the blood is one of the standard indicators for diagnosing that there is some coagulopathy associated with the coronavirus disease [14]. Lungs and vascular ultrasonography enable us to determine the severity of the disease. Both of these procedures play a very important role in intensive care unit $[15,16]$. Both of these have major advantages over other imaging techniques (irradiation, CT scan, etc.).

\section{Vascular ultrasonography}

Vascular ultrasonography is a non-invasive, remarkably versatile procedure. It has several advantages over other imaging techniques including no exposure to radiation. There is also no need for an injection of a contrast dye, to which a small percentage of people are allergic. Zero chance of allergic reaction is one of the biggest advantages of vascular ultrasonography [17]. No risks are reported while using Doppler ultrasonography. This procedure is so safe that it can be used in pregnant women and no risks have been reported which can cause damage to the growing fetus. Doppler ultrasonography doesn't involve any needles or any anesthesia so chances of a traumatic reaction are reduced to minimal. The mechanics of vascular ultrasonography is based on the principle proposed by Doppler which is termed as Doppler Effect. "The Doppler Effect" or "Doppler Shift' is termed as the change in frequency noted by the observer who is moving towards or away from the source that is generating the wave. So if the object is moving towards the observer he will note an increase in frequency and if the object is moving away from the observer, a decrease in frequency will be observed. Doppler ultrasound is used to diagnose conditions in which the blood flow to a tissue is reduced or completely obstructed. For example, in coronary artery disease, vessels supplying to the heart are completely or partially occluded.

There are different types of Doppler ultrasonography tests that are being used nowadays. Each of the types has its own pros and cons. They include the following
- Power Doppler: This type is used when a very small size of tissue has to be interrogated and it provides more details about that tissue than Color Doppler.

- Color Doppler: In this type, sound waves of different frequencies are converted to different colors by the use of computers. By using color Doppler ultrasonography, we can determine the direction as well as the flow rate of the blood.

- Spectral Doppler: Spectral Doppler presents the findings in the form of graphical representation. It shows how much of the lumen of the blood vessel is occluded.

- Continuous Doppler: As the name suggests, sound waves are sent and received in a continuous manner. This type is more accurate than the other and has the advantage of studying blood that is flowing at a faster rate

\section{How does vascular ultrasonography work?}

The principle that can be applied to do the blood flowing in the vessels is determined by placing a transducer over the vessels. The blood cells are in constant motion in the body moving from one site to other emitting waves of different frequencies. The red blood cells that are moving towards the probe will exhibit higher frequency indicating the direction of the flow of blood. In vascular ultrasonography, sound waves are pitched upon the tissue which is being interrogated and they are reflected from the constantly moving cells inside the vessels. These reflections in a sound wave are measured by the machine an image is produced. We can also determine the flow rate of the moving blood. The flow rate helps us in determining whether a thrombus or stenosed artery is present which will disrupt the laminar flow in the vessels to turbulent flow. The normal flow in a blood vessel is represented by the presence of clear windows and thin waveform as opposed to thickened waveform in the absence of clear windows. The term that is used to represent turbulent flow in ultrasonography is called "spectral broadening".

There are two scanning planes in vascular ultrasonography. The first is the transverse plane in which the transducer is perpendicular to the surface of the blood vessel so the image that is formed is in the shape of a circle as a result of the position of the transducer. This shows the cross-section of the blood vessel. The second plane is the longitudinal plane. In this plane, the transducer is parallel to the surface of the blood vessel and the image formed is in the shape of a rectangle. One of the most important steps in vascular ultrasonography is directing the beam at a specific angle on the vessel. The angle between the beam and the vessel should be less than 60 degrees. If the angle is not correct, there is a good chance that the resulting image will alter our results. The blood vessels in our body have characteristic waveforms. The blood flow in a vessel is dependent upon two factors which are the 
pressure differential and the resistance offered by the walls of the vessel. The pressure differential is dependent upon the cardiac function and also the position of the vessel in the circulatory system while the resistance relies mainly on the need of the tissue which the blood vessel is supplying. In a normal aorta, the thickness of the waveform should be very low which indicates a smooth pattern of blood flow. With the help of waveform, we can determine the direction, velocity, and acceleration of blood inside the vessels.

\section{Coagulation disorders associated with Covid-19}

Coronavirus 19 is spreading worldwide and is causing an increase in the death rate day by day everywhere it goes [18]. The economies of most countries have taken a steep slope downhill because of this virus. Since December 2019, this virus has affected more than 86.2 million people and has caused 1.87 million deaths around the globe. Coronavirus belongs to a large family of common viruses that are associated with respiratory disorders and are found both in humans as well as animals. The novel coronavirus 2019 shares very similar characteristics to the coronavirus pandemic in 2002 [19]. The word "Çorona"' 'is a Latin word that's been assigned to this virus because of its structure. There are protein spikes that are present all over the body of this virus giving it a crown-like appearance. The coronavirus is a single-stranded RNA virus and four structural proteins that are common to all species of viruses that belong to the family of Coronavirus namely Spike(S), Membrane (M), Envelope (E), and Nucleocapsid protein $(\mathrm{N})$. Each of these proteins plays a vital role in the pathogenicity of the virus.

The spike protein helps the virus in recognition and binding to Angiotensin converting enzyme receptors that are present in the heart, kidney, and lungs of the host cells [20]. The $M$ protein is the most abundant structural protein found in the coronavirus and it defines the shape and also maintains the interaction between the other structural proteins [21]. The envelope protein is the smallest and it helps the viral genome in the process of integration in the whole self and also its release. The synergistic action of membrane and envelope proteins helps this virus to turn the host cell into a factory for the synthesis of its genome [22]. Lastly, the nucleocapsid proteins are present just underneath the surface proteins and encapsulate the whole of the viral genome [23, 24]. These proteins are derived from the host and make a layer that is fatty in nature. This is the reason why hand washing with soap is so encouraged these days because it disrupts the very nature of the protective layer surrounding the viral genome.

\section{Pathogenesis}

Many coagulopathies are being associated with the coronavirus 19 when the disease is in its most severe form. These complications include disseminated intravascular coagulation, arterial thrombosis, pulmonary embolism, sepsis-induced coagulopathy, and deep venous thrombosis, all of which are being observed in the severe cases of coronavirus. The coronavirus gains its entry by using the angiotensin converting enzyme receptor which is acted upon by angiotensin converting enzyme [25]. This receptor is present in many organs in the body but the most abundant site for this receptor is the lungs. Other organs that possess these receptors are the heart, kidneys, intestines, and arteries. The endothelial cells present in the walls of the blood vessel are lined by angiotensin converting enzyme receptors. The virus enters the receptors and these receptors are then endocytosed. Through the process of endocytosis, the virus gains entry $^{26}$. Upon its arrival, the virus activates the endothelial cells and expresses the adhesion molecules like VCAM-1 and ICAM-1 [27, 28]. These molecules help in the adhesion and activation of leukocytes. The virus also increases the activation of Von Willebrand factor and P-selectin alongside tissue factor [29]. The tissue factor makes a complex with factor VII and starts the process of coagulation. Tissue factor is also secreted by monocytes causes the increased production of microthrombi in the vasculature of the lungs causing diseases like pulmonary embolism. Another protein secreted by monocytes is called Alpha thrombin which is a key enzyme in fibrin generation and also is a potent activator of platelets hence being a source of disturbances in the microvasculature of the body [30].

Recent analysis regarding the thrombotic events in patients suffering from coronavirus suggests that the venous thromboembolic events are present in only those patients in which the disease takes at least 17 to 25 days to resolve after the onset. The clinical signs of acute pulmonary embolism are chest pain, dyspnea, hemoptysis, and sometimes presyncope. These symptoms are masked in the Corona patients because a continuous supply of vasopressors and supplemental $\mathrm{O} 2$ is being given to the patients. These are the reasons which pose a challenge for the clinicians to employ imaging techniques like duplex venous ultrasonography.

The diagnosis of vascular anomalies in patients suffering from coronavirus is dependent on the increasing levels of D-dimer, increased prothrombin time, and thrombocytopenia. D-dimer is one of the two products formed when a clot is broken after being acted upon by plasmin, the activated form of plasminogen. The other product is named FDP (fibrin degradation products). The presence of elevated levels of D-dimers indicates that many clots are being broken down which is a characteristic of acute respiratory distress syndrome, pulmonary embolism, or deep venous thrombosis [31].

\section{Vascular ultrasonography in Coronavirus-19 \\ In patients suffering from severe cases of coronavirus disease, coagulopathies like arterial}


thrombosis, disseminated intravascular coagulation, deep venous thrombosis, and pulmonary embolism have a higher incidence and these complications later become the cause of death in these patients [32, 33]. When a vessel is stenosed, there are changes in the flow of blood upstream and downstream from the narrowing of the vessel. The resistive index is a term that signifies the resistance of a vascular bed that lies distal to the part of the vessel that is being interrogated. So when the lumen of the vessel is narrowed due to any reason, for example, atherosclerosis, thrombus, or embolus, there is an increase in the resistive index of the vessel along with the blood pressure and blood flow. The ultra sonogram demonstrates this as a rise in the resistive index upstream of the vessel narrowing. At the site of the stenosis, the sonogram exhibits an increase in the peak systolic velocity. This increase in velocity is because of the reason that the same amount of blood has to pass through the vessel whose radius is now decreased. Downstream from the site of thrombosis or stenosis, the resistive index is decreased because that part of the tissue is hungry for more blood and its vascular resistance is decreased to help in compensating the decreased blood flow. The presence of turbulent flow is seen distal to the stenosis or thrombosis in the sonogram. This turbulent flow is represented by spectral broadening with a slow upstroke and no clear windows. We characterize an upstroke as a slow upstroke when the time to reach a peak is greater than 70 milliseconds on the waveform.

To detect deep venous thrombosis, various imaging techniques are required [34]. Clinical assessment is not sufficient enough to diagnose deep venous thrombosis in the early stages. Various imaging techniques like using radioisotope studies ( ${ }^{125}$ fibrinogen, $\mathrm{I}^{111}$ labeled platelet), Doppler ultrasonography, plethysmography, elevated D-dimer levels [35], thrombocytopenia, are used for DVT diagnosis [36]. Studies conducted to detect the authenticity of this test showed that it has a sensitivity of 71 to 95 percent and specificity was found to be 72 to 97 percent. Recently, the position of this test as the most authentic one in diagnosing the coagulopathies associated with coronavirus was overthrown by another test called duplex venous ultrasonography. Both the sensitivity and specificity of duplex venous ultrasonography are much more than impedance plethysmography which is the reason why duplex venous ultrasonography is replacing all other imaging techniques.

There are four sites on the body where the transducer is placed for the recording is done while performing duplex venous ultrasonography. First is the posterior tibial vein behind the medial malleolus. The second is the popliteal vein behind the knee. The third is the superficial femoral vein and the fourth is the common femoral vein at the groin. During this procedure, multiple segments of veins are interrogated.
If the compression is applied distal to the thrombus, an increase in the flow of blood is seen as the stagnant blood is pushed towards the heart. If the compression is applied proximal to the occluded area, no change in the flow is seen. So with the help of compressions, we can determine the location of the thrombus. There is also a risk of false-negative tests in duplex venous ultrasonography which can occur as a consequence of non-obstructive and obstructive clots in one of the paired vessels. This alteration in the result can be avoided by taking two-dimensional imaging. The veins are interrogated in both the transverse and longitudinal planes to minimize the chance of the result being falsenegative.

\section{Treatment}

Many abnormalities like pulmonary embolism, arterial thrombosis, disseminated intravascular coagulation, deep vein thrombosis are detected only when the Coronavirus disease progression is in its last stages and when the mortality rate associated with the disease becomes very high. Vascular ultrasonography helps us in making early interventions so that the number of severe cases can be reduced to a minimum. There is no specific antiviral treatment and a vaccine produced still in phase 3 of the drug trial but if the coagulopathies are detected in the early stages of viral infection, the chances of survival are increased manyfolds.

Heparin is given to patient who is an anticoagulant which prevents the formation of clot hence decreasing the number of clots formed in deep venous thrombosis or disseminated intravascular coagulation. A recent paper suggested that the use of heparin has reduced the mortality rate in corona patients [37]. One of the major side effects of using heparin is heparin-induced thrombocytopenia or HIT. Acute respiratory distress syndrome is one of the commonest complications associated with tissue factor mediated thrombin generation in coronavirus [38]. Low molecular weight heparin or Fondaparinux are currently being used. Employment of these drugs is useful as their major advantage is the reduced risk of heparininduced thrombocytopenia.

Protease inhibitors and P2Y12 inhibitors are also used $[39,40]$. Protease inhibitors are used in a very calculated amount when given with drugs like clopidogrel, prasugrel because some of the protease inhibitors like ritonavir decrease the level of active metabolites of P2Y12 inhibitors causing a decline in their efficacy. Some clinical studies have also suggested the benefits of tranexamic acid. This drug suppresses the conversion of plasminogen to plasmin. Although plasmin is used to break the clots already formed in the body, there is a considerable amount of evidence that suggests that the use of plasmin increases the infectivity of viruses by cleaving a protein in its genome [41]. 


\section{CONCLUSION}

Undoubtedly, Corona virus has disturbed the very balance of the whole world, affecting millions of lives and influencing every aspect of life. The economies of some countries have been demolished. Scientists are working day and night to get their hands on as much information as they can get on the progression of the disease so new and more effective methods can be devised to help us win the war against this troublesome disease. Till now, we are in a very much better place as compared to when the first case of Coronavirus was reported in Wuhan. Still, a lot of mysteries are yet to be resolved and the mechanism through which this virus causes its pathological features is one of the hottest topics in the field of medicine. Maximum efforts are being employed in hindering the disease to reach its maximum potential ultimately causing a decline in the mortality rate. Several complications are associated with Coronavirus-19 out of which vascular abnormalities hold their ground as one of the most significant pathologies alongside acute respiratory distress syndrome, acidosis, inflammation, and kidney failure. Amongst many other techniques, vascular ultrasonography helps us in identifying the different coagulopathies that may occur in conjunction with the disease and ultimately enabling us to intervene in the initial stages of progression of the disease. The use of ultrasonography has markedly increased over the past few years because of its significant role in detecting diseases in their early stages.

\section{REFERENCES}

1. Baloch, S., Baloch, M. A., Zheng, T., \& Pei, X. (2020). The coronavirus disease 2019 (COVID-19) pandemic. The Tohoku journal of experimental medicine, 250(4), 271-278.

2. Khan, M., Khan, H., Khan, S., \& Nawaz, M. (2020). Epidemiological and clinical characteristics of coronavirus disease (COVID-19) cases at a screening clinic during the early outbreak period: a single-centre study. Journal of medical microbiology, 69(8), 1114.

3. Liu, K., Zhang, W., Yang, Y., Zhang, J., Li, Y., \& Chen, Y. (2020). Respiratory rehabilitation in elderly patients with COVID-19: A randomized controlled study. Complementary therapies in clinical practice, 39, 101166.

4. Vakili, S., Savardashtaki, A., Jamalnia, S., Tabrizi, R., Nematollahi, M. H., Jafarinia, M., \& Akbari, H. (2020). Laboratory findings of COVID-19 infection are conflicting in different age groups and pregnant women: a literature review. Archives of medical research.

5. Hu, Y., Sun, J., Dai, Z., Deng, H., Li, X., Huang, Q., ... \& Xu, Y. (2020). Prevalence and severity of corona virus disease 2019 (COVID-19): A systematic review and meta-analysis. Journal of clinical virology, 104371.

6. Zhu, N., Wang, W., Liu, Z., Liang, C., Wang, W., Ye, F., ... Tan, W. (2020). Morphogenesis and cytopathic effect of SARS-CoV-2 infection in human airway epithelial cells. Nature Communications, 11(1).

7. Wang, H. J., Du, S. H., Yue, X., \& Chen, C. X. (2020). Review and Prospect of Pathological Features of Corona Virus Disease. $\mathrm{Fa}$ yi xue za zhi, 36(1), 16-20.

8. Hon, K. L., Leung, K. K. Y., Leung, A. K., Sridhar, S., Qian, S., Lee, S. L., \& Colin, A. A. (2020). Overview: The history and pediatric perspectives of severe acute respiratory syndromes: Novel or just like SARS. Pediatric pulmonology, 55(7), 1584-1591.

9. Iba, T., Levy, J. H., Levi, M., Connors, J. M., \& Thachil, J. (2020). Coagulopathy of coronavirus disease 2019. Critical care medicine.

10. Pascarella, G., Strumia, A., Piliego, C., Bruno, F., Del Buono, R., Costa, F., ... \& Agrò, F. E. (2020). COVID- 19 diagnosis and management: a comprehensive review. Journal of internal medicine, 288(2), 192-206.

11. Allinovi, M., Parise, A., Giacalone, M., Amerio, A., Delsante, M., Odone, A., ... \& Mangia, A. (2020). Lung ultrasound may support diagnosis and monitoring of COVID-19 pneumonia. Ultrasound in Medicine and Biology, 46(11), 2908-2917.

12. Sun, X., Wang, T., Cai, D., Hu, Z., Liao, H., Zhi, L., .. \& Wang, A. (2020). Cytokine storm intervention in the early stages of COVID-19 pneumonia. Cytokine \& growth factor reviews, 53 , $38-42$.

13. Li, X., Xu, S., Yu, M., Wang, K., Tao, Y., Zhou, Y., ... \& Zhao, J. (2020). Risk factors for severity and mortality in adult COVID-19 inpatients in Wuhan. Journal of Allergy and Clinical Immunology, 146(1), 110-118.

14. Zhang, L., Yan, X., Fan, Q., Liu, H., Liu, X., Liu, Z., \& Zhang, Z. (2020). D- dimer levels on admission to predict in- hospital mortality in patients with Covid- 19. Journal of Thrombosis and Haemostasis, 18(6), 1324-1329.

15. Schmid, M., Escher, F., \& Clevert, D. A. (2020). Lung ultrasonography in COVID-19 pneumonia. Der Radiologe, 60(10), 919-926.

16. Plesner, L. L., Dyrberg, E., Hansen, I. V., Abild, A., \& Andersen, M. B. (2020). Billeddiagnostiske fund ved COVID-19. Ugeskr Lager, 182, V03200191.

17. Yang, L., \& Ran, H. (2018). Extracranial vertebral artery dissection: Findings and advantages of ultrasonography. Medicine, 97(9).

18. Malik, Y. A. (2020). Properties of coronavirus and SARS-CoV-2. The Malaysian journal of pathology, 42(1), 3-11.

19. Hasöksüz, M., Kiliç, S., \& Saraç, F. (2020). Coronaviruses and sars-cov-2. Turkish journal of medical sciences, 50(SI-1), 549-556.

20. Li, F. (2016). Structure, function, and evolution of coronavirus spike proteins. Annual review of virology, 3, 237-261. 
21. Alsaadi, E. A., \& Jones, I. M. (2019). Membrane binding proteins of coronaviruses. Future Virology, 14(4), 275.

22. Yoshimoto, F. K. (2020). The proteins of severe acute respiratory syndrome coronavirus-2 (SARS CoV-2 or n-COV19), the cause of COVID-19. The protein journal, 39, 198-216.

23. Srinivasan, S., Cui, H., Gao, Z., Liu, M., Lu, S., Mkandawire, W., ... \& Korkin, D. (2020). Structural genomics of SARS-CoV-2 indicates evolutionary conserved functional regions of viral proteins. Viruses, 12(4), 360.

24. O’Leary, V. B., Dolly, O. J., Höschl, C., Černa, M., \& Ovsepian, S. V. (2021). Unpacking Pandora From Its Box: Deciphering the Molecular Basis of the SARS-CoV-2 Coronavirus. International journal of molecular sciences, 22(1), 386.

25. Shang, J., Wan, Y., Luo, C., Ye, G., Geng, Q., Auerbach, A., \& Li, F. (2020). Cell entry mechanisms of SARS-CoV-2. Proceedings of the National Academy of Sciences, 117(21), 1172711734.

26. Wang, S., Guo, F., Liu, K., Wang, H., Rao, S., Yang, P., \& Jiang, C. (2008). Endocytosis of the receptor-binding domain of SARS-CoV spike protein together with virus receptor ACE2. Virus research, 136(1-2), 8-15.

27. Hayden, M. R. (2020). Endothelial activation and dysfunction in metabolic syndrome, type 2 diabetes and coronavirus disease 2019. Journal of International Medical Research, 48(7), 0300060520939746.

28. Amraei, R., \& Rahimi, N. (2020). COVID-19, renin-angiotensin system and endothelial dysfunction. Cells, 9(7), 1652.

29. Bautista-Vargas, M., Bonilla-Abadía, F., \& Cañas, C. A. (2020). Potential role for tissue factor in the pathogenesis of hypercoagulability associated with in COVID-19. Journal of thrombosis and thrombolysis, 50, 479-483.

30. Colling, M. E., \& Kanthi, Y. (2020). <? covid19?> COVID-19-associated coagulopathy: An exploration of mechanisms. Vascular Medicine, 25(5), 471-478.

31. Schutgens, R. E. (2020). D-dimer in COVID-19: A Guide With Pitfalls. HemaSphere, 4(4).
32. Zamboni, P. (2020). COVID-19 as a vascular disease: lesson learned from imaging and blood biomarkers.

33. Giannis, D., Ziogas, I. A., \& Gianni, P. (2020). Coagulation disorders in coronavirus infected patients: COVID-19, SARS-CoV-1, MERS-CoV and lessons from the past. Journal of Clinical Virology, 127, 104362.

34. Bernardi, E., \& Camporese, G. (2018). Diagnosis of deep-vein thrombosis. Thrombosis research, 163, 201-206.

35. Sartori, M., Cosmi, B., Legnani, C., Favaretto, E., Valdré, L., Guazzaloca, G., ... \& Palareti, G. (2012). The Wells rule and D-dimer for the diagnosis of isolated distal deep vein thrombosis. Journal of thrombosis and haemostasis, 10(11), 2264-2269.

36. Evaluation of Quantitative Impedance Plethysmography for Continuous Blood Flow Measurement: I. Electrode Systems;

37. Ayerbe, L., Risco, C., \& Ayis, S. (2020). The association between treatment with heparin and survival in patients with Covid-19. Journal of thrombosis and thrombolysis, 50, 298-301.

38. Li, X., \& Ma, X. (2020). Acute respiratory failure in COVID-19: is it "typical" ARDS?. Critical Care, 24, 1-5.

39. Kumar, D., Kumari, K., Vishvakarma, V. K., Jayaraj, A., Kumar, D., Ramappa, V. K., ... \& Singh, P. (2020). Promising inhibitors of main protease of novel corona virus to prevent the spread of COVID-19 using docking and molecular dynamics simulation. Journal of Biomolecular Structure and Dynamics, 1-15.

40. Ahsan, W., Javed, S., Al Bratty, M., Alhazmi, H. A., \& Najmi, A. (2020). Treatment of SARS-CoV2: How far have we reached?. Drug discoveries \& therapeutics.

41. Waqas, U., Waseem, N., ALI, A., \& Nadeem, S. (2020). In Vivo Analysis of Therapeutic Effects of Hydroxychloroquine, Azithromycin, Paracetamol, Dexamethasone, Remdesivir and Tocilizumab in Patients Affected with Novel Covid-19. In Vivo, 81 . 